Errata

\title{
Character Representation
}

Harry E. Gaylord

Vakgroep Alfa Informatica, Rijsuniversiteit Groningen, POB 716, Groningen 9700 AS, The Netherlands e-mail: galiard@let.rug.nl

Computers and the Humanities 29: 51-73, 1995.

On pages 68,69 and 71 , the wrong figure captions were pages show the corrected versions. Kluwer Academic printed for figures 7,8 , and 10 . The following three Publishers regret these errors. 


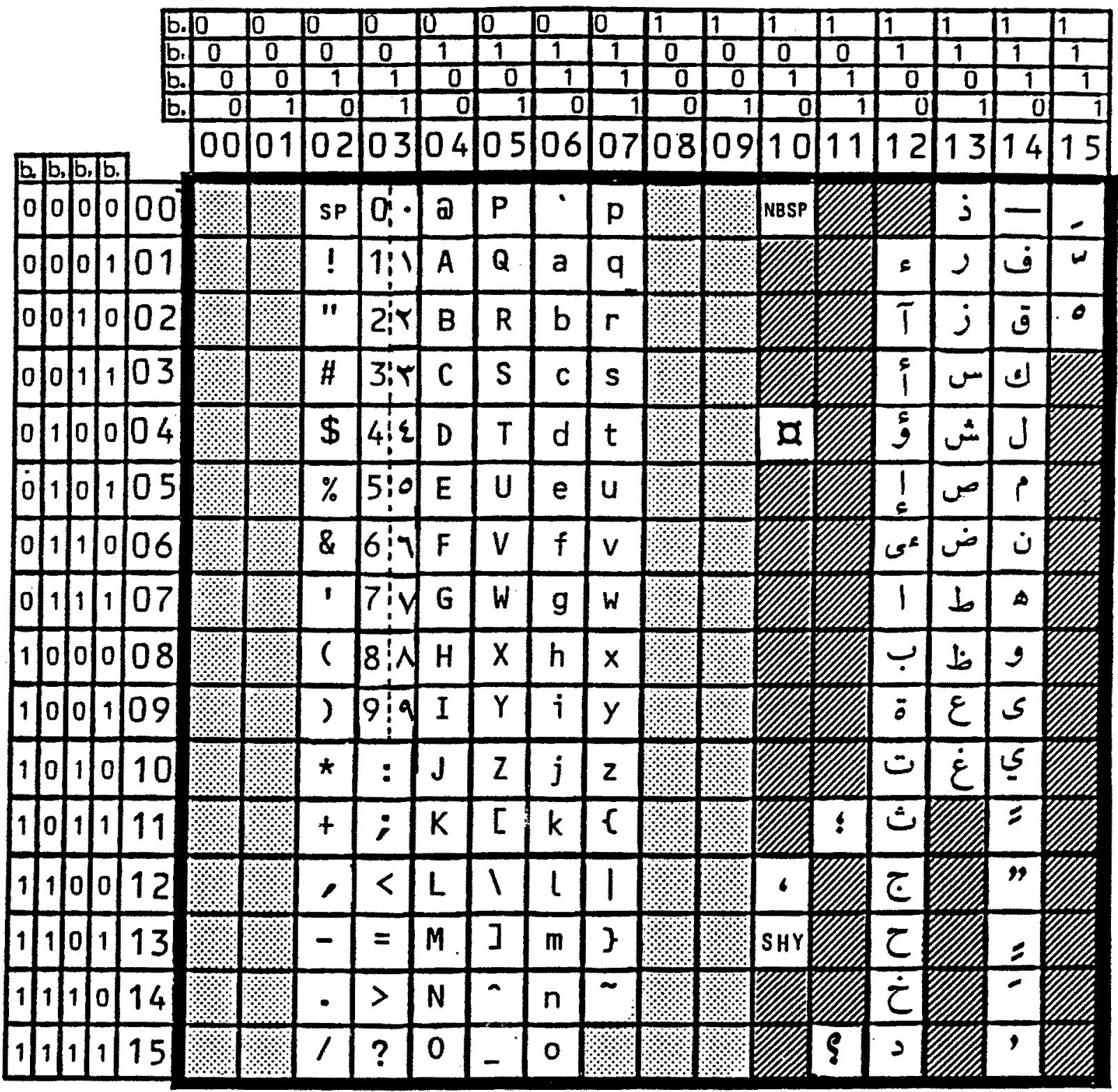

Fig. 7. ISO 8859-6 Latin/Arabic. 


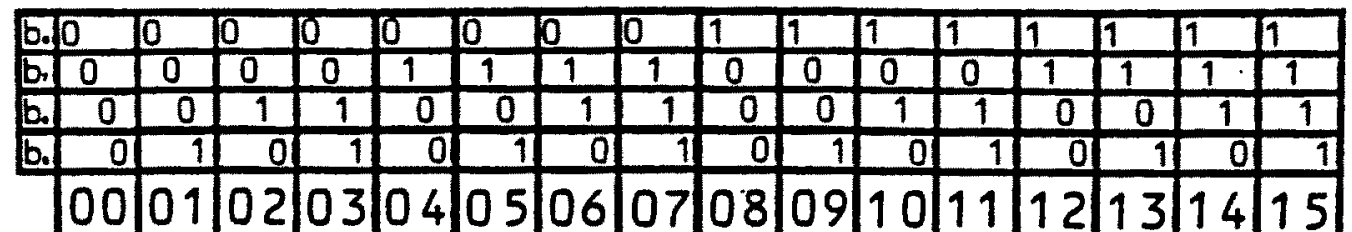

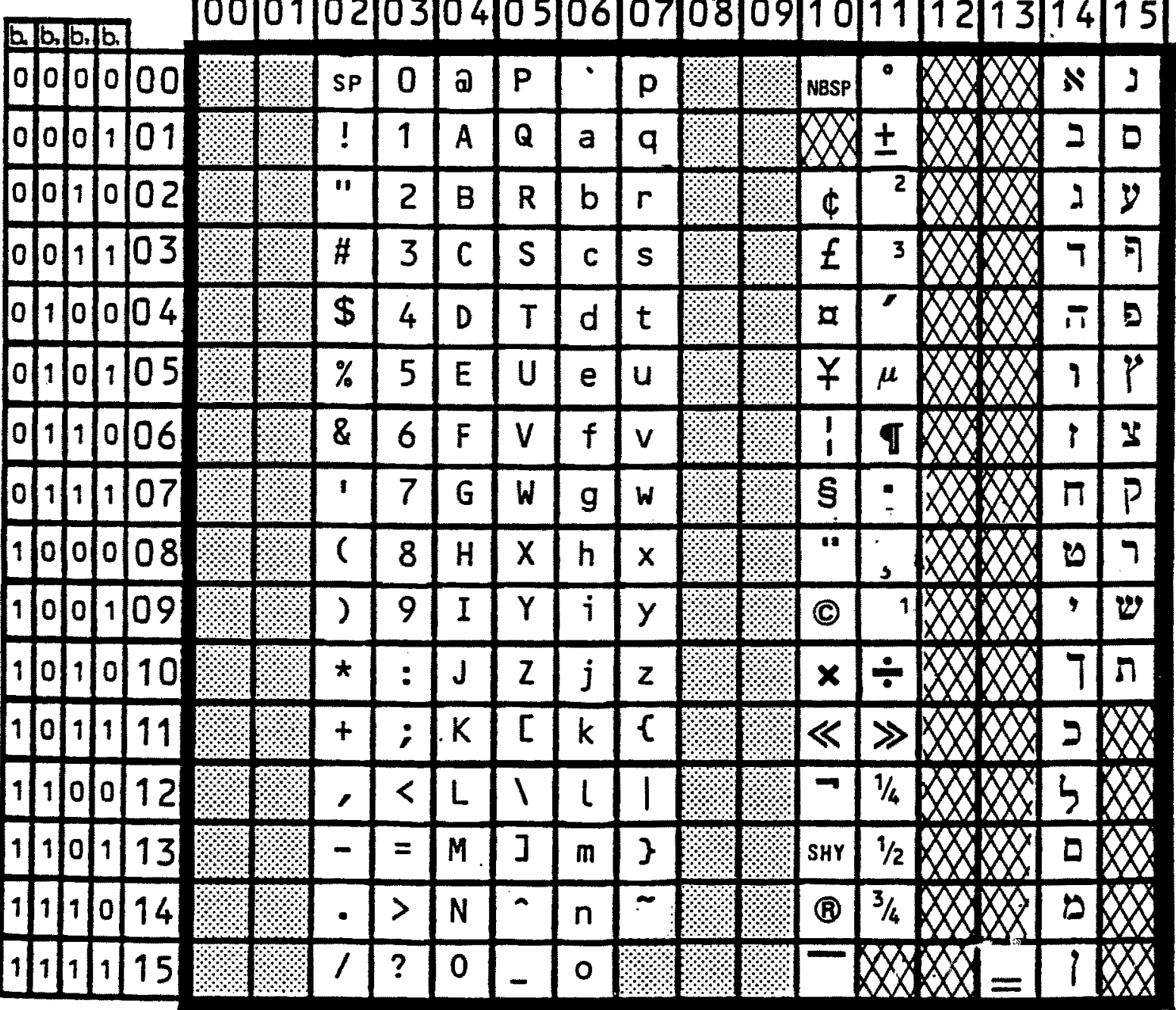

Fig. 8. ISO 8859-8 Latin/Hebrew. 


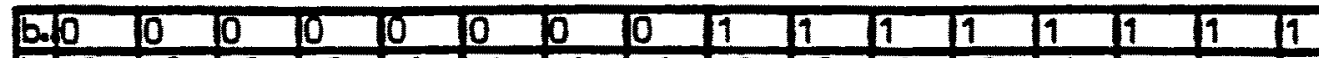
\begin{tabular}{llllllllllllllllllllll}
\hline b. & 0 & 0 & 0 & 0 & 2 & 1 & 1 & 1 & 0 & 0 & 0 & 0 & 1 & 1 & 1 & 1 \\
\hline
\end{tabular} \begin{tabular}{lllllllllllllllll|l|l|l|l|}
\hline B & 0 & 0 & 1 & 1 & 0 & 0 & 1 & 1 & 0 & 0 & 1 & 1 & 0 & 0 & 1 & 1 \\
\hline
\end{tabular} \begin{tabular}{llllllllllllllllll}
\hline 6 & 0 & 1 & 0 & 1 & 0 & 1 & 0 & 1 & 0 & 1 & 0 & 1 & 0 & 1 & 0 & 1 \\
\hline
\end{tabular}

\begin{tabular}{|c|c|c|c|c|c|c|c|c|c|c|c|c|c|c|}
\hline 0 & 1000 & & $S P$ & 0 & a & $P$ & & p & & 0 & 0 & & $\theta$ & \\
\hline & \begin{tabular}{l|l|l}
1 & 1
\end{tabular} & & $!$ & 1 & $\sqrt{A}$ & $Q$ & $a$ & $\bar{q}$ & & $\bar{A}$ & a & & $\bar{N}$ & \begin{tabular}{l|l}
$\dot{a}$ & 1
\end{tabular} \\
\hline & 002 & & $"$ & 2 & $B$ & $R$ & $b$ & $r$ & & $\overline{\bar{E}}$ & $\bar{e}$ & $\hat{\hat{A}}$ & $\overline{0}$ & \begin{tabular}{l|l} 
ât \\
\end{tabular} \\
\hline & 1103 & & $\#$ & 3 & c & $s$ & $c$ & $s$ & & $G$ & $\bar{g}$ & $\bar{A}$ & ól & ã \\
\hline & 10104 & & $\$$ & 4 & $D$ & $T$ & $d$ & $t$ & & $\overline{\mathrm{I}}$ & 7 & $A^{\prime}$ & ô & $a ̈: c$ \\
\hline & 1105 & & $\%$ & 5 & $E$ & $U$ & $e$ & $u$ & & \begin{tabular}{|l|l|}
$\mathbf{I}$ \\
\end{tabular} & 7 & A & $\tilde{0}$ & $\bar{a} \cdot \bar{c}$ \\
\hline 1 & 0106 & & 8 & 6 & $F$ & $\mathrm{v}$ & $f$ & $v$ & & $\mathrm{~K}$ & ks & $\bar{E}$ & $\overline{0}$ & $\bar{x} \bar{x}$ \\
\hline & 1.07 & & 1 & 7 & $G$ & $w$ & $g$ & $w$ & & $\mathbf{\xi}$ & $\cdot$ & I & $\overline{0}$ & \begin{tabular}{l|l}
$\mathrm{i}$ & $\mathrm{i}$
\end{tabular} \\
\hline & 10108 & & 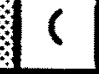 & 8 & $\mathrm{H}$ & $x$ & $h$ & $x$ & & 4. & \} & $\check{c}$ & $\emptyset$ & $\check{c} \mid l$ \\
\hline & 1109 & & ) & 9 & I & $Y$ & $i$ & $y$ & & $\theta$ & वा & $\hat{E}$ & $\overline{\mathrm{U}}$ & ély \\
\hline & \begin{tabular}{|l|l|l|}
0 & 10 \\
\end{tabular} & & $\star$ & $\because$ & $\mathrm{J}$ & $z$ & $j$ & $z$ & & $\check{\mathbf{s}}$ & $\bar{s}$ & $\bar{E}$ & Ú & $\bar{e}$ \\
\hline 1 & \begin{tabular}{|l|l|}
1 & 11 \\
\end{tabular} & & + & $;$ & $K$ & {[} & $k$ & l & & $\mp$ & 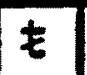 & $\ddot{\mathrm{E}}$ & $\hat{U}$ & $\vec{e} \mid \hat{c}$ \\
\hline 10 & $\begin{array}{lll}0 & 12 \\
\end{array}$ & &. & $<$ & $L$ & 1 & 1 & 1 & & $\bar{Z}$ & $\bar{z}$ & $\dot{\mathrm{E}}$ & نे & $\dot{\mathrm{e}} \mathbf{c}$ \\
\hline 问 & $\begin{array}{lll}1 & 13 \\
\end{array}$ & & - & $=$ & $\bar{M}$. & $\mathrm{J}$ & $m$ & 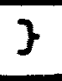 & & \begin{tabular}{|l|} 
SHY \\
\end{tabular} & -1 & $\dot{I}$ & $\bar{Y}$ & i \\
\hline & \begin{tabular}{l|l|l|l|l}
0 & 14
\end{tabular} & & $\cdot 1$ & 18 & $\mathrm{~N}$ & • & $n$ & & & $\bar{U}$ & $\bar{u}$ & $\overline{\hat{I}}$ & $\bar{\beta}$ & $\hat{\mathfrak{\jmath}}$ \\
\hline & 1115 & & 1 & & & & & & & $\mathbb{N}$ & & & $B$ & $\overline{\mathbf{i}}$ \\
\hline
\end{tabular}

